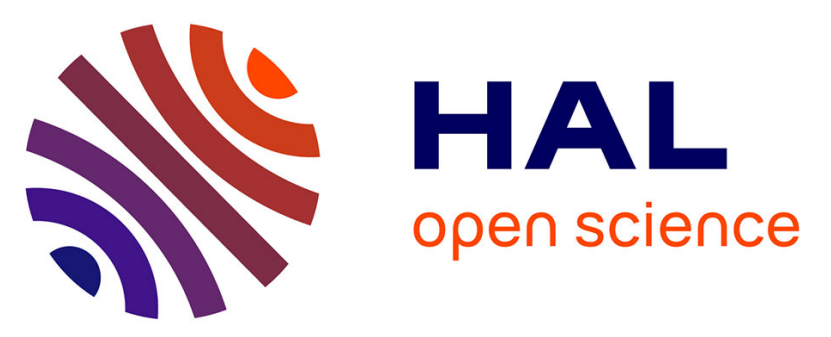

\title{
High pressure-high temperature synthesis of diamond from single-wall pristine and iodine doped carbon nanotube bundles
}

\author{
A. Merlen, Pierre Toulemonde, S. Le Floch, G. Montagnac, Tahar \\ Hammouda, O. Marty, A. San Miguel
}

\section{To cite this version:}

A. Merlen, Pierre Toulemonde, S. Le Floch, G. Montagnac, Tahar Hammouda, et al.. High pressurehigh temperature synthesis of diamond from single-wall pristine and iodine doped carbon nanotube bundles. Carbon, 2009, 47, pp.1643-1651. 10.1016/j.carbon.2009.02.014 . hal-00453379

\author{
HAL Id: hal-00453379 \\ https://hal.science/hal-00453379
}

Submitted on 19 Nov 2021

HAL is a multi-disciplinary open access archive for the deposit and dissemination of scientific research documents, whether they are published or not. The documents may come from teaching and research institutions in France or abroad, or from public or private research centers.
L'archive ouverte pluridisciplinaire HAL, est destinée au dépôt et à la diffusion de documents scientifiques de niveau recherche, publiés ou non, émanant des établissements d'enseignement et de recherche français ou étrangers, des laboratoires publics ou privés.

\section{(ㅇ)(1) $\$$}

Distributed under a Creative Commons Attribution - NonCommerciall 4.0 International 


\title{
High pressure-high temperature synthesis of diamond from single-wall pristine and iodine doped carbon nanotube bundles
}

\author{
A. Merlen*,a,b,c, P. Toulemonde ${ }^{\mathrm{a}, \mathrm{d}}$, S. Le Floch ${ }^{\mathrm{a}}$, G. Montagnac ${ }^{\mathrm{e}}, \mathrm{T}$. \\ Hammouda ${ }^{\mathrm{f}}$, O. Marty ${ }^{\mathrm{g}}$, A. San Miguel ${ }^{* *, a}$ \\ ${ }^{a}$ Université de Lyon, F-69622, Lyon, France; Université Lyon 1, Villeurbanne; CNRS \\ UMR5586, Laboratoire de Physique de la Matière Condensée et Nanostructures \\ ${ }^{b}$ Université du Sud Toulon-Var, IM2NP, France. \\ ${ }^{c}$ CNRS, IM2NP (UMR 6242) Bâtiment R, BP 132, F-83957 La Garde Cedex, France. \\ ${ }^{d}$ Institut Néel, CNRS and Université Joseph Fourier, 25 avenue des Martyrs, BP 166, \\ F-38042 Grenoble cedex 9, France. \\ ${ }^{e}$ Laboratoire de Sciences de la Terre, UMR 5570 CNRS-ENSL-UCBL, 46 Allée d'Italie, \\ F-69364 Lyon Cedex 0\%, France \\ ${ }^{f}$ Laboratoire Magmas et Volcans CNRS UMR6524, Observatoire de Physique du Globe \\ de Clermont Ferrand, Université Blaise Pascal 5, rue Kessler,63038 Clermont-Ferrand \\ cedex, France \\ ${ }^{g}$ INL, Université Claude Bernard Lyon-1,69622 Villeurbanne, France.
}

\begin{abstract}
High pressure and high temperature experiments were performed on single wall carbon nanotube bundles up to $14.5 \mathrm{GPa}$ and $1800 \mathrm{~K}$. Depending on the thermodynamic conditions we have observed three different behaviors: at ambient temperature and for pressure lower than 24 GPa minor structural changes are observed. Depending on the loss of hydrostatic conditions or on the combined application of pressure and temperature, partial or total graphitization is observed. For pressures of $14.5 \mathrm{GPa}$ and temperatures of $1800 \mathrm{~K}$ the nanotubes are irreversibly transformed into cubic diamond, show-
\end{abstract}

\footnotetext{
*corresponding author, Fax: +33-494142168, merlen@univ-tln.fr

** corresponding author, Fax: +33-472432648, sanmigue@lpmcn.univ-lyon1.fr
} 
ing that it is possible to synthesize under high pressure and high temperature pure $\mathrm{sp}^{3}$ carbon structures from single wall carbon nanotubes. In the case of iodine intercalated nanotubes, the same conditions of $14.5 \mathrm{GPa}$ and $1800 \mathrm{~K}$ lead also to the transformation into diamond. No evidence of incorporation of iodine in the $\mathrm{sp}^{3}$ carbon structure was found. On the basis of our results we discuss possibilities for new carbon-carbon composite engineering from single wall carbon nanotube bundles.

\section{Introduction}

The synthesis of new carbon $\mathrm{sp}^{3}$ structures constitutes a real challenge in view of some of their expected promising properties as extremely high hardness $(1 ; 2)$ or high critical temperature superconductivity $(3 ; 4)$. Many theoretical works propose different types of such structures $(5 ; 6 ; 7 ; 8 ; 9 ; 10)$ including nano-cage based ones. Since the synthesis of industrial diamond in the $50 \mathrm{~s}(11)$ it is well known that high pressure and high temperature (HP$\mathrm{HT}$ ) is a relevant process for the synthesis of $\mathrm{sp}^{3}$ carbon structures as well as other $\mathrm{sp}^{3}$ based ultra-hard materials as cubic BN. As regards diamond synthesis the precursor used for such synthesis is generally graphite, and the use of metallic solvents leads to an important lowering of the pressure and temperature conditions of synthesis. Without the presence of catalizers, the crystal structure of graphite remains unchanged after the compression up to $15 \mathrm{GPa}$ at room temperature and loses some of the graphite features at higher pressure(12). It has been also reported recently (13) the reversible high pressure transformation of graphite to a superhard phase which could be based of a mixed $\mathrm{sp}^{2}-\mathrm{sp}^{3}$ carbon hybridization. Direct conversion from polycrystalline 
graphite to diamond at room temperature and quasi-hydrostatic conditions has been observed above $70 \mathrm{GPa}(14)$. The transformation of graphite to other forms of $\mathrm{sp}^{2}$ carbon as nano-onions in non-hydrostatic conditions have been also reported(15). A preferred transformation to the metastable hexagonal diamond form (lonsdaleite) appears to depend of many factors as temperature or the degree of desorganization of the initial $\mathrm{sp}^{2}$ carbon form. The discovery of the $\mathrm{sp}^{2}$ based nano-forms of carbon as fullerenes or carbon nanotubes, brought new opportunities for the elaboration of new $\mathrm{sp}^{3}$ carbon based structures. Intermediate carbon $\mathrm{sp}^{2}-\mathrm{sp}^{3}$ materials exhibiting exceptional mechanical properties have been obtained through the high pressure and high temperature treatment of $\mathrm{C}_{60}$ fullerenes $(16 ; 17 ; 18)$. Several reviews have appeared discussing the high-pressure and high-temperature phase diagram of $\mathrm{C}_{60}$ and we refer the reader to recent ones $(19 ; 20 ; 21)$ and references therein for more details. The mechanism governing the formation of such structures is the multiple-direction polymerization of the fullerenes through carbon cycloaddition, i.e., the formation of $\mathrm{sp}^{3}$ bonds between the carbon molecules. Different morphologies can also be obtained through high pressure and high temperature treatment of nanostructured carbon. In that way, Dubrovinskaia et al (22) synthesized carbon nanorods from $\mathrm{C}_{60}$ fullerenes treated at $20 \mathrm{GPa}$ and $\mathrm{T}=2500 \mathrm{~K}$ in a multi-anvil cell. The obtained material, constituted of crystals of $20 \mathrm{~nm}$ width and lengths up to $1 \mu \mathrm{m}$ showed a bulk modulus of $491 \mathrm{GPa}$ which surpass the typical diamond value of $442 \mathrm{GPa}$. Since their first observation in 1991 carbon nanotubes have been also considered as a potential precursor material for the HP-HT synthesis of new carbon forms. As for fullerenes, their structure can be considered as close to $\mathrm{sp}^{2}$ hy- 
bridized. Among all different carbon nanotubes types, single wall carbon nanotubes (SWCNT) are particular candidates for HP-HT synthesis precursors as the mechanism of cycloaddition predicted by theoretical calculations for transition towards pure $\mathrm{sp}^{3}$ structures could be strongly facilitate by their structural organization in bundles. Such feature has lead to many theoretical $(7 ; 8 ; 23 ; 24 ; 25 ; 26 ; 27)$ and experimental $(28 ; 29 ; 30 ; 31 ; 32 ; 33 ; 34)$ works concerning their high pressure evolution. Some of these works suggest that it could be possible through high pressure application to form intertube bonds in a pure $\mathrm{sp}^{3}$ carbon structure $(7 ; 8)$. Nevertheless a reaction phase diagram for SWCNT under pressure and high temperature similar to the ones proposed for $\mathrm{C}_{60}$ fullerenes is still unknown and few experimental studies on this subject have been performed up to now $(35 ; 31 ; 36 ; 37)$. Different reasons can be evoked to explain such disparity with respect to the fullerene case. First of all the dependence of the mechanical properties of carbon nanotubes on their diameter which will imply a diameter dependent reaction diagram. A second and important reason is the relatively low crystallinity of nanotube samples. In fact, in most samples, SWCNT bundles are typically constituted of 20 to 50 tubes, which snake between other bundles without structural organization at that level. Finally, depending on the synthesis method, the distribution of carbon nanotubes can be peaked around a certain diameter or on the contrary exhibit a broad size distribution. All these factors should be integrated in the discussion of the P-T evolution of carbon nanotubes and constitute real difficulties for the development of a SWCNT phase diagram. The transformation towards diamond at high pressure and high temperature has been reported in several works for multiwalled carbon 
nanotubes (MWCNT). Cao et al.(38) synthesized diamond from MWCNT 20 to $50 \mathrm{~nm}$ in diameter at $4.5 \mathrm{GPa}$ and $1600 \mathrm{~K}$ in the presence of NiMnCo solvent. MWCNT heated in a diamond anvil cell by a $\mathrm{CO}_{2}$ laser above 17 GPa and $2500 \mathrm{~K}$ transformed to nano-sized octahedral diamond crystals of less than $50 \mathrm{~nm}$ (39). Purified MWCNT, without the presence of solvent, show to be stable at thermodynamic conditions between 5.5 and $6.5 \mathrm{GPa}$ and temperatures of 1150-1250 K(40). In the case of SWCNT, even if transformation to phases exhibiting exceptional mechanical properties have been described, up to our knowledge, there is no report on their transformation towards the diamond phase. Wang et al(41) showed that SWCNT could be transformed at $75 \mathrm{GPa}$ and ambient temperature in a new hexagonal carbon structure with a bulk modulus comparable to diamond. Popov et al(42) on their side showed that with strong non hydrostatic pressure, a superhard phase can be obtained at $24 \mathrm{GPa}$ (hydrostatic component of a stress tensor) and ambient conditions. The observation of new superhard phases obtained at high pressure and non hydrostatic conditions was confirmed by other groups(43). Few experiments combining high pressure and high temperature on SWCNT have been also reported. From $1.5 \mathrm{GPa}$ and $1000 \mathrm{~K}$, Khabashesku et al(35) observed a modification of the microstructure morphology of SWCNT. The experiments, performed in uniaxial type presses, lead to the formation of micrometer size flakes, which transformed back to the original morphology after sonication. In their work, for experiments at 8 GPa and temperatures between 800 and $1500 \mathrm{~K}$, polyhedral (hexagonal, cubic, and rhombohedral) nano- and micro- scale size crystallites were observed. The electron diffraction images obtained for similar crystallites formed at 9.5 
GPa and temperatures between 700 and $900 \mathrm{~K}$ gave rise to cubic or hexagonal symmetries with cell parameters differing from the cubic or hexagonal diamond ones. Finally, they observed the total disappearance of the Raman RBM (radial breathing mode) of their $1.2 \mathrm{~nm}$ diameter tubes at $8 \mathrm{GPa}$ and $1500 \mathrm{~K}$ and the transformation of the sample to nanographite forms at 8 $\mathrm{GPa}$ and $1800 \mathrm{~K}$. The $\mathrm{sp}^{2} / \mathrm{sp}^{3}$ characterization of the observed crystallites was not possible in that work. Kawasaki et al(31) treated at high pressure and high temperature SWCNT of similar diameters to ours and observed an important increase of hardness in the recovered samples when they were submitted to a treatment above $11 \mathrm{GPa}$ and $773 \mathrm{~K}$. This was interpreted as the formation of $\mathrm{sp}^{3}$ bonds in the tubes, that was not observed for samples treated at $8 \mathrm{GPa}$ and $773 \mathrm{~K}$ or at $13 \mathrm{GPa}$ and $473 \mathrm{~K}$. In this paper we report experimental work performed on pristine and iodine doped SWCNT under high pressure and high temperature conditions. The stability of SWCNT and their potential as precursor material for the elaboration of new HP-HT $\mathrm{sp}^{3}$ forms of carbon is examined. In particular, the synthesis of diamond from SWCNT is demonstrated and the potential incorporation of iodine in the structure explored.

\section{Experiments}

We performed high pressure experiments on SWCNT using different thermodynamic conditions. Three different kinds of nanotubes were used: two pristine and one doped. Pristine SWCNT nanotubes from MER corporation (arc discharge synthesis) were characterized showing a high degree of purity and good cristallinity with a size of bundles of around 50 tubes in average. 
The pristine SWCNT from Nanoledge corporation (arc discharge synthesis) had bundles of 20 tubes in average and did not followed any purification process and hence included the presence of traces of the Ni catalyst. Both kinds of samples have nanotubes of $1.3 \pm 0.2 \mathrm{~nm}$ diameter, organized in bundles. Due to the purification process SWCNT from $M E R$ are shorter and are open-ended tubes. The last sample we used was iodine doped SWCNT. The iodine doping process of the nanotubes is described elsewhere(44) and was done on the Nanoledge tubes batch.

The three samples were submitted to high pressure and high temperature conditions seeking for their eventual irreversible structural evolution after HP-HT cycle. Three different high pressure apparatus were used. The highest pressures (up to $25 \mathrm{GPa}$ ) were performed using a diamond anvil cell (DAC) with different pressure transmitting media (argon, 4:1 methanol ethanol or paraffin oil) which assure quasi-hydrostatic conditions. Nevertheless, as mentioned in previous works, the choice of this medium has a strong effect on the evolution of SWCNT $(45 ; 34)$.

For HP-HT experiments we used two different presses. In the toroidal press (Paris-Edinburgh press) the sample is encapsuled in a boron epoxy gasket and compressed by two opposite sintered diamond anvils (46). In this case, the volume of the sample is higher, but due to the absence of pressure transmitting medium, uniaxial stress components can play a role. Near the sample we used two stripes of rhenium which constitute the resistive heater to reach a temperature of $1500 \mathrm{~K}$ under a pressure of $12 \mathrm{GPa}$. Pressure and temperature were evaluated from the press calibration(46).

Finally, the last experiments were performed by using the French na- 
tional 1000-ton multi-anvil facility in Clermont-Ferrand, France. Experimental conditions and pressure calibrations were similar to those described previously(47).In this case the sample is encapsuled in a platinum crucible and $\mathrm{MgO}$ is used as pressure transmitting medium. $\mathrm{LaCrO}_{3}$ is used as heating element and the temperature is measured by a thermocouple. Pressure is determined with a calibration curve previously obtained with well known phase transitions.

For all HP-HT experiments the protocol was almost the same: high pressure was progressively applied (typical increasing speed: 1 hour/GPa). At the maximum pressure reached high temperature was applied during one (ParisEdinburgh press) or two hours (multianvil press). Then the pressure was slowly decreased to avoid damage of the anvils (8-10 hours for decompression using the multi-anvil press).

After each experiment, the recovered samples were studied by Raman spectroscopy, which is known to be a powerful technique to detect structural modifications in carbon structures. Scanning and transmission electron microscopy were also performed.

\section{Results}

First of all we performed high pressure experiments on pristine $M E R$ SWCNT using a diamond anvil cell. The results are reported in figure 1. The sample was compressed up to $19 \mathrm{GPa}$ or $24 \mathrm{GPa}$ using different pressure transmitting media. In the two experiments, the characteristic nanotube Raman modes, i.e., the radial breathing modes (RBM) at low frequencies and the G-band at high energies are observed after the high pressure cycle. We 
note nevertheless, that the overall intensity has strongly decreased, as it can be appreciated by the lower signal to noise ratio, and also that the full half width maximum of the G-band has slightly increased. In these experiments, the $\mathrm{D}$ band was not visible due to the presence of the strong Raman peak of the diamond anvil.

High pressure and high temperature (HP-HT) experiments in the ParisEdinburgh press were performed using Nanoledge SWCNT. The Raman spectra before and after HP-HT experiments are reported in figure 2. The sole application of pressure up to $12 \mathrm{GPa}$ leads to a strong attenuation of the Raman signal and a much stronger G-band enlargement than what it was observed using the diamond anvil cell at higher pressures. The absence of pressure transmitting medium which leads to less hydrostatic conditions can be considered as the most probable origin for this observation. We also note that some modifications take place in the RBM in the spectrum recovered from $12 \mathrm{GPa}$, but the Notch filter cutoff of the Raman spectroscopy set-up is too close to the signal for a quantitative analysis. When a temperature of $1500 \mathrm{~K}$ is applied under a pressure of $12 \mathrm{GPa}$ the changes in the Raman spectra are much more important: the RBM have totally disappeared, the intensity of the $\mathrm{D}$ band is much more important and the characteristic doublet of G-band is strongly modified. On the right part of figure 2 are shown TEM pictures of recovered samples after HP or HP-HT treatment. For the HPHT sample we observed graphene planes characteristic of disordered graphite (interlayer spacing ranging from $0.36 \mathrm{~nm}$ and $0.39 \mathrm{~nm}$ ), confirming Raman experiments. For the sample having followed solely a HP cycle, the TEM 
images did not allow to observe the presence of nanotubes even if the RBM signal in Raman spectra suggest that a fraction of the original nanotubes have been preserved. The TEM images show also the presence of distorted graphene planes suggesting that part of the nanotubes have been transformed to disordered graphite.

In the above reported experiments we have not found any clear signature pointing out to the synthesis of $\mathrm{sp}^{3}$-based structures. Thus we performed HP-HT experiments in a multi-anvil press using more drastic thermodynamic conditions. In the case of Nanoledge pristine SWCNT the Raman spectrum of the recovered sample after $14.5 \mathrm{GPa}$ and $1800 \mathrm{~K}$ treatment is shown on the left panel of figure 3. We observed only one sharp Raman peak for this sample: the characteristic $T_{2 g} 1331 \mathrm{~cm}^{-1}$ mode of cubic diamond, showing that it is possible to perform diamond HP-HT synthesis from pristine SWCNT. The typical size of the diamond crystallites is $0.5-1 \mu \mathrm{m}$ as can be seen on the SEM picture in figure 3.

The last experiment here reported concerns iodine intercalated SWCNT as precursor material. For this sample the presence of host atoms inside the nanotubes structure, confirmed in some recent studies (44), could prevent the transition under HP-HT towards diamond and lead to the synthesis of cage like materials, just like observed in the case of silicon clathrates (48). The experiments were performed using a multi-anvil press at the same thermodynamic conditions as pristine SWCNT: $14.5 \mathrm{GPa}$ and $1800 \mathrm{~K}$. In these conditions the main features of the recovered sample are reported on figure 
4. As regards its Raman spectra (left part of figure 4) we observed only one Raman mode whose frequency seems to be highly sensitive to laser beam power: for a laser power around $0.3 \mathrm{~mW}$ we observed only one peak at 1331 $\mathrm{cm}^{-1}$ corresponding to the $T_{2 g}$ diamond mode. This mode is shifted towards lower energy when the laser power is increased up to $3 \mathrm{~mW}$. In the inset of the Raman spectra shown in figure 4 we can see that this shift can vary between 0 and $15 \mathrm{~cm}^{-1}$ depending on the power used and the sampled area. Moreover, the sample seems to be inhomogeneous: some parts are highly sensitive to laser power whereas some other are not affected. The SEM picture in figure 4 confirms that the size of the diamond crystallites is broadly distributed between $10 \mu \mathrm{m}$ and less than $0.1 \mu \mathrm{m}$. The chemical analysis by EDX spectroscopy indicates the presence of iodine with a very small proportion of platinum (which should be due to the crucible) and nickel (coming from the residual impurities of the catalyst used for the commercial preparation of SWCNT). The TEM observation confirms the high disparity in size of the crystallites. All inspected directions in the High Resolution TEM pictures do not indicate any distortion in the diamond network (figure 4). The elemental analysis by EDX spectroscopy of the crystallites observed by TEM indicates that there is no iodine atoms inside diamond crystallites, suggesting that iodine atoms have diffused during synthesis and are localized at the grain boundaries.

Table I summarizes the main characteristics and results of the 6 performed experiments. 


\section{Discussion}

The above experiments allow us to discuss the effect of high pressure and high temperature conditions on the stability of bundled SWCNT and the possibility of synthesis of new $\mathrm{sp}^{3}$-based carbon structures.

Let us first discuss the results from the experiments at ambient temperature. Up to the maximum reached pressure of $24 \mathrm{GPa}$, the Raman signal of the recovered samples at ambient pressure preserve the main features of the Raman spectra. In the two different SWCNT samples studied (from Nanoledge or MER) the RBM and G-band are still present in the Raman spectra after the pressure cycle suggesting that at least part of the nanotubes have not been destroyed by high pressure application. Nevertheless we do note that the intensity of the D-band can grow after the pressure cycle (Fig 2 ). This has been also observed during the pressurization of nanotubes and has been correlated to the onset of structural defaults or new structures in nanotubes depending on the used pressure transmitting medium(45;34). In the case of the absence of pressure transmitting medium, the D-band grow is correlated to a very strong enlargement of the G-band coupled to observation of an important proportion of graphitic forms. We can then conclude on the fundamental role of hydrostatic conditions on the pressure stability of SWCNT bundles in close agreement with the observation of superhard phases obtained under strong deviatoric stress(49; 43). At pressures of $12 \mathrm{GPa}$ with no pressure transmitting medium both nanotubes and graphitic forms coexist in our sample. As it has been pointed out(34), the transformation of tubes of larger diameter are probably associated with such transformation, as the collapse transition is predicted to scale with the third inverse power 
of the tube diameter.

We can now consider the combined effect of pressure and temperature on SWCNT bundles. Under thermodynamic conditions of $12 \mathrm{GPa}$ and $1500 \mathrm{~K}$ we observe strong structural changes on the recovered samples as confirmed by Raman spectroscopy. The TEM images point out to the sample graphitization. Nevertheless, the similarity between our Raman spectra and those reported by Khabashesku et al (35) suggests that a partial polymerization of nanotubes could occur for such HP-HT conditions.

For more drastic HP-HT conditions SWCNT are irreversibly transformed into diamond showing that it is possible to synthesize under HP-HT $\mathrm{sp}^{3}$ carbon structure using single-wall carbon nanotubes as precursor material, as it has been reported from multiwall carbon nanotubes(50).

Sumiya et al(51) have recently shown that the onset temperature for polycrystalline diamond formation from graphitic or non graphitic forms of carbon is $\mathrm{P} \geq 15 \mathrm{GPa}$ and $1800-1900 \mathrm{~K}$. Nevertheless, these authors found that the required temperature conditions for pure polycrystalline diamond formation are $\mathrm{T} \geq 2400 \mathrm{~K}$ for graphite and $\mathrm{T} \geq 1900 \mathrm{~K}$ for non-graphitic carbon. In their work, they explained such differences due to a different mechanism of synthesis: the polycrystalline diamond will be formed due to the diffusion process plus a a two-step martensitic process for graphitic precursors, whereas polycrystilline diamond would be synthesized only through a diffusion process without graphitization or formation of intermediate phases from non-graphitic carbon as carbon nanotubes. We find that carbon nanotubes can be totally transformed to microcrystalline diamond at $14.5 \mathrm{GPa}$ and $1800 \mathrm{~K}$ in close agreement with Sumiya et al. On the other side, we 
find that for samples submitted to $12 \mathrm{GPa}$ and $1500 \mathrm{~K}$, SWCNT graphitize. The formation of diamond from SWCNT could be then mediated through an intermediate step involving graphitization, in contrast with the proposition of Sumiya et al.

Considering recent studies on the pressure behavior of carbon nanotubes bundles, we can propose a scenario for their HP-HT transformation which is sketched in Fig 5. We have recently shown, in close agreement to calculations that SWCNT bundles follow two major modifications(34). The first one, taking place at low pressures, involves the continuous modification of the nanotube cross-section. This modification takes place at pressures between 1 and 3 GPa depending on the nanotube diameter and it has been discussed in many previous works. At higher pressure, the collapse of the nanotubes is observed, involving the flattening and approaching of the nanotubes wall. Both transitions can be reversible, but for the higher diameter tubes an irreversibly graphitization after the collapse has been observed. The pressure at which evidence of the collapse transition is observed, strongly depends on the pressure transmitting medium(34). For silicon oil pressure transmitting medium (PTM) the collapse transition is observed at approximately $15 \mathrm{GPa}$ a value that increases for PTM showing higher hydrostaticity. Our observations are compatible with a collapse transition at pressures between 10 and 12 GPa with no PTM showing partial graphitization that it is associated with the larger diameter tubes. With increasing temperature total graphitization is reached at temperatures below $1500 \mathrm{~K}$. Thermodynamic conditions of 14.5 GPa and $1800 \mathrm{~K}$ lead to diamond formation. This is depicted in Fig 5a for a bundle of nanotubes of having equal diameter, large enough to allow graphi- 
tization before diamond formation. As already mentioned, the mechanical instability of carbon nanotubes is known to strongly depend on the nanotube radius with a dependence close to the $1 / R^{3}$. Consequently, it should be reasonable to expect that for nanotubes with smaller radious, conditions for $\mathrm{s} p^{3}$ bonding can be reached at HP-HT before the collapse plus graphitization of the tubes takes place. This situation will favor the formation of nanotubes bundles in which the nanotubes are assembled via $\mathrm{sp}^{3}$ bonds. This situation which has been never observed, is depicted in Fig 5b. Differences of chirality inside the bundle and non favorable bond orientation, will probably lead to a lack of spatial coherence of such assemblage as shown in Fig 5b. To support this idea, it is interesting to confront the present data on the stability of carbon nanotubes with those existing for other carbon nanostructures. The best studied case is $\mathrm{C}_{60}$ fullerenes. Under HP-HT conditions, crystals of pristine $\mathrm{C}_{60}$ 's follow a series of phase transformation including the formation of $1 \mathrm{D}, 2 \mathrm{D}$ or $3 \mathrm{D}$ polymers which have been extensively studied $(16 ; 52 ; 53 ; 19 ; 20 ; 21 ; 54)$. One of the main conclusion of these works was that high temperature is essential to induce such polymerization transitions of $\mathrm{C}_{60}$ under pressure, even if it is possible to induce collapse of fullerenes towards diamond at ambient temperature in highly uniaxial compression (55). This point is not surprising comparing fullerenes and nanotubes structure: for both structures it is necessary to afford good orientation of carbon bonds to assure the cyclo-addition responsible of the intertube bonds formation and high temperature offers such possibility.

Finally, real samples are made of nanotubes having a distribution of sizes. 
Two situations are then possible. In the first one, such distribution is done inside the bundles themselves. In that case, a very interesting situation could be obtained following Fig 5c. The transformation into diamond of the larger tubes could be frustrated by the presence of small diameter nanotubes, leading to the formation at HP-HT of carbon-carbon nanocomposite made of $\mathrm{s} p^{3}$ assembled nanotubes in a graphitic like matrix, which could also exhibit a high degree of $\mathrm{sp}^{3}$ bonding. The second possibility would be a sample constituted of a mixture of bundles having different diameters, but with no distribution of nanotube diameter inside each bundle. In that case, HPHT conditions could lead to a mixture of the situations of Fig 5a and 5b, i.e., a composite made of nanometric diamond crystals and assembled tubes nanocrystal exhibiting a very low crystallinity degree as explained.

All these possibilities confer to SWCNT an enormous potential for the engineering of new carbon composites which should exhibit very interesting mechanical properties. In addition, the possibility of including donor or acceptor atoms in the nanotube structures allows, in principle, to expect the electrical tuning of these materials. In fact, as we have seen, iodine appears to segregate from diamond, but we could reasonable think that iodine atoms could be preserved in nanotubes with smaller diameters. Another interesting path is the development of such HP-HT approach in other types of nanotubes as $\mathrm{BN}$ single wall nanotubes. In fact, it has been shown from calculations that the tube collapse and $\mathrm{sp}^{3}$ bonding can be also induced by pressure application(56).

The diameter and chirality distribution in bundled SWCNT is then a key point to approach its HP-HT phase diagram. Depending on the nan- 
otube size, graphitization can take place before the thermodynamic conditions needed for intertube bond formation are reached. This appears to be our case. In such case, diamond formation is favored from the resulting graphitic forms. On the other side a strong nanotube diameter distribution would allow to preserve the smaller tubes and then favor the formation of carbon-carbon composites. Only those nanotubes bundles made of tubes with such small diameter - which could prevent collapse before reaching the thermodynamic conditions favoring $\mathrm{sp}^{3}$ formation - would be good candidates for $\mathrm{sp}^{3}$ networking at HP-HT conditions. Further experimentation or calculations will be needed to start to develop the basis of such phase diagrams and the consequent elaboration of new carbon materials following HP-HT conditions.

\section{Summary and conclusion}

We have reported HP-HT experiments performed on pristine and iodine doped SWCNT in different thermodynamic conditions and with different experimental set-ups. Comparing our experiments and other works we have proposed a scenario of transformation of SWCNT bundles which include the collapse of the nanotubes followed by partial or total (thermally activated) graphitization and the formation of diamond at higher pressures. With similar thermodynamic conditions, iodine intercalated SWCNT also transforms into diamond and no sign of iodine incorporation in the diamond lattice was observed. As a final point, we point out that the engineering of new carbon materials could be favored by the use of smaller diameter nanotubes or even the use of samples with a wide diameter distribution. 


\section{Acknowledgments}

The authors are indebted to Dr. E. D. Obraztsova and Pr. J. L. Sauvajol for fruitful discussions. This work was supported by the French Ministère Délégué à la Recherche et aux Nouvelles Technologies and the CNRS through grants "ACI-2003 NR0122" and "ACI-2003 JC2077". The multianvil apparatus of Laboratoire Magmas et Volcans is financially supported by the Centre National de la Recherche Scientifique (Instrument national de IINSU).

\section{References}

[1] X. Blase, P. Gillet, A. San-Miguel, P. Mélinon, Exceptional ideal strength of carbon clathrates, Phys. Rev. Lett. 2004; 92(21): 215505215508.

[2] N. Rey, A. Múnoz, P. Rodríguez-Hernández, A. San-Miguel, Firstprinciples study of lithium-doped carbon clathrates under pressure, J. of Phys. Cond. Mat. 2008; 20: 215218-215224.

[3] D. Connétable, V. Timoshevskii, B. Masenelli, J. Beille, J. Marcus, B. Barbara, A. M. Saitta, G. M. Rignanese, P. Mélinon, S. Yamanaka, X. Blase, Superconductivity in doped $\mathrm{sp}^{3}$ semiconductors: The case of the clathrates, Phys. Rev. Lett. 2003; 91(24): 247001-247004.

[4] X. Blase, C. Adessi, D. Connétable, Role of the dopant in the superconductivity of diamond, Phys. Rev. Lett. 2004; 93(23): 237004-237007. 
[5] S. Fahy, S. Louie, High-pressure structural and electronic properties of carbon, Phys. Rev. B 1987; 36(6): 3373-3385.

[6] D. Yildirim, O. Gulseren, C. Klc, S. Ciraci, Pressure-induced interlinking of carbon nanotubes, Phys. Rev. B 2000(19); 62: 12648-12651.

[7] L. Chernozatonskii, E. Richter, M. Menon, Crystals of covalently bonded carbon nanotubes: Energetics and electronic structures, Phys. Rev. B 2002; 65(24): 241404(R)-241407.

[8] S. Reich, C. Thomsen, P. Ordejon, Elastic properties and pressure induced phase transitions of single-walled carbon nanotubes., phys. stat. sol. (b) 2003; 235(1): 354-359.

[9] S. F. Braga, D. S. Galvao, Single wall carbon nanotubes polymerization under compression: An atomistic molecular dynamics study, Chem. Phys. Lett. 2006; 419(4-6): 394-399.

[10] F. Ribeiro, P. Tangney, S. Louie, M. L. Cohen, Hypothetical hard structures of carbon with cubic symmetry, Phys. Rev. B 2006; 74(17): 172101-172104.

[11] F. Bundy, H. Hall, H. Strong, R. J. Wentorf, Man-made diamonds 1955; Nature 176: 51-55.

[12] F. Bundy, W. Bassett, M. Weathers, R. Hemley, H. Mao, A. Goncharov, The pressure-temperature phase and transformation diagram for carbon; updated through 1994, Carbon 1996; 34(2): 141-153. 
[13] W. L. Mao, H. K. Mao, P. J. Eng, T. P. T. M. Newville, C. C. Kao, D. L. Heinz, J. Shu, Y. Meng, J. Shu, R. J. Hemley., Bonding changes in compressed superhard graphite, Science 2003; 302: 425-427.

[14] T. Schindler, Y. Vohra, A micro-Raman investigation of high-pressure quenched graphite, J. Phys.: Condens. Matter 1995; 55(7): L637-L642.

[15] V. Blank, V. Denisov, A. Kirichenko, B. Kulnitskiy, S. Y. Martushov, B. Mavrin, I. Perezhogin, High pressure transformation of single-crystal graphite to form molecular carbononion, Nanotechnology 2007; 18(34): 345601.

[16] M. Nunez-Regueiro, L. Marques, J. Hodeau, O. Bethoux, M. Perroux, Polymerized fullerite structures, Phys. Rev. Lett. 1995; 74(2): 278-281.

[17] V. Blank, M. Popov, G. Pivovarov, N. Lvova, K. Gogolinsky, V. Reshetov, Ultrahard and superhard phases of fullerite $\mathrm{C}_{60}$ : comparison with diamond on hardness and wear, Diamond and Related Materials 1998; 7(2-5): 427-431.

[18] S. Yamanaka, N. Kini, A. Kubo, S. Jida, H. Kuramoto, Topochemical $3 \mathrm{D}$ polymerization of $\mathrm{C}_{60}$ under high pressure at elevated temperatures, JACS 2008; 130(13): 4303-4309.

[19] B. Sundqvist, Fullerenes under high pressures, Adv. in Phys. 1999; 48(1): $1-134$.

[20] R. Moret, Structures, phase transitions and orientational properties of the $\mathrm{C}_{60}$ monomer and polymers, Acta Cryst. 2005; A61: 62-76. 
[21] A. San-Miguel, Nanomaterials under high pressure, Chem. Society Reviews $2006 ; 35(10)$ : 876-889.

[22] N. Dubrovinskaia, L. Dubrovinsky, W. Crichton, F. Langenhorst, A. Richter, Aggregated diamond nanorods, the densest and least compressible form of carbon, App. Phys. Lett. 2005; 87(8): 083106.

[23] S.-P. Chan, W.-L. Yim, X. G. Gong, Z.-F. Liu, Carbon nanotube bundles under high pressure: transformation to low-symmetry structures, Phys. Rev. B 2003; 68(7): 075404-075414.

[24] J. A. Elliott, J. K. W. Sandler, A. H. Windle, R. J. Young, M. S. P. Shaffer, Collapse of single-wall carbon nanotubes is diameter dependent, Phys. Rev. Lett. 2004; 92 (9): 095501-095504.

[25] J. Zang, A. Treibergs, Y. Han, F. Liu, Geometric constant defining shape transitions of carbon nanotubes under pressure, Phys. Rev. Lett. 2004; 92(10): 105501-105504.

[26] M. H. F. Sluiter, Y. Kawazoe, Phase diagram of single-wall carbon nanotube crystals under hydrostatic pressure, Phys. Rev. B 2004; 69(22): 224111-224121.

[27] M. H. F. Sluiter, V. Kumar, Y. Kawazoe, Symmetry-driven phase transformations in single-wall carbon-nanotube bundles under hydrostatic pressure, Phys. Rev. B 2002; 65(16): 161402(R)-161405.

[28] I. Loa, Raman spectroscopy on carbon nanotubes at high pressure, J. of Raman Spectroscopy 2003; 34(7-8): 611-627. 
[29] S. Rols, I. N. Gontcharenko, R. Almairac, J. L. Sauvajol, I. Mirebeau, Polygonization of single-wall carbon nanotube bundles under high pressure, Phys. Rev. B 2001; 64(15): 153401-153404.

[30] S. M. Sharma, S. Karmakar, S. K. Sikka, P. V. Teredesai, A. K. Sood, A. Govindaraj, C. N. R. Rao, Pressure-induced phase transformation and structural resilience of single-wall carbon nanotube bundles, Phys. Rev. B 2001; 63(20): 205417-205421.

[31] S. Kawasaki, Y. Matsuoka, A. Yao, H. Touhara, K. Suito, High pressure behavior of single-walled carbon nanotubes and polymerized fullerenes, J. Phys. Chem. Sol. 2004; 65(2-3): 327-331.

[32] A. Merlen, N. Bendiab, P. Toulemonde, A. Aouizerat, A. San Miguel, J.-L. Sauvajol, G. Montagnac, H. Cardon, P. Petit, Resonant Raman spectroscopy of single-wall carbon nanotubes under pressure, Phys. Rev. B 2005; 72(3): 035409-035414.

[33] P. T. C. Freire, V. Lemos, J. A. Lima, G. D. Saraiva, P. S. Pizani, R. O. Nascimento, N. M. P. S. Ricardo, J. Mendes, A. G. Souza, Pressure effects on surfactant solubilized single-wall carbon nanotubes, phys. stat. sol. b 2007; 244(1): 105-109.

[34] C. Caillier, D. Machon, A. San-Miguel, R. Arenal, G. Montagnac, H. Cardon, M. Kalbac, M. Zukalova, L. Kavan, Probing high-pressure properties of single-wall carbon nanotubes through fullerene encapsulation, Phys. Rev. B 2008; 77(12): 125418-125426. 
[35] V. N. Khabashesku, Z. Gu, B. Brinson, J. L. Zimmerman, J. L. Margrave, V. A. Davydov, L. S. Kashevarova, A. V. Rakhmanina, Polymerization of single-wall carbon nanotubes under high pressures and high temperatures, J. Phys. Chem. B 2002; 106(43): 11155-11162.

[36] S. Kawasaki, Y. Matsuoka, T. Yokomae, Y. Nojima, F. Okino, H. Touhara, H. Kataura, Effect of a liquid pressure-transmitting medium on the high pressure behavior of open- and closed-end single-walled carbon nanotubes and of $\mathrm{C}_{60}$-peapods, Phys. stat. sol. b 2004; 241(15): 3512-3516.

[37] S. Kawasaki, Y. Nojimab, T. Yokomaeb, F. Okinob, H. Touhara., Hardness of high-pressure high-temperature treated single-walled carbon nanotubes, Physica B 2007; 388(1-2): 59-62.

[38] L. Cao, C. Gao, H. Sun, G. Zoub, Z. Zhang, X. Zhang, M. He, M. Zhang, Y. Li, J. Zhang, D. Dai, L. Sun, W. Wang, Synthesis of diamond from carbon nanotubes under high pressure and high temperature, Carbon 2001; 39(2): 287-290.

[39] H. Yusa, Nanocrystalline diamond directly transformed from carbon nanotubes under high pressure, Diamond and Related Materials 2002; 11(1): 87-91.

[40] M. Corrias, P. Serpa, P. Kalck, G. Dechambre, J. Lacout, C. Castiglioni, Y. Kihn, H igh purity multiwalled carbon nanotubes under high pressure and high temperature, Carbon 2003, 41(12): 2361-2367. 
[41] Z. Wang, Y. Zhao, K. Tait, X. Liao, D. Schiferl, C. Zha, R. Downs, J. Qian, Y. Zhu, T. Shen, A quenchable superhard carbon phase synthesized by cold compression of carbon nanotubes, PNAS 2004; 101(38): 13699-13702.

[42] M. Popov, M. Kyotani, Y. Koga, Superhard phase of single wall carbon nanotube: comparison with fullerite $\mathrm{C}_{60}$ and diamond, Diamond and Related Materials 2003; 12(3-7): 833-839.

[43] V. Blank, V. Denisov, A. Kirichenko, N. Lvova, S. Martyushov, B. Mavrin, D. Popova, M. Popov, E. Tatyanin, A. Zakhidov, Nanostructured superhard carbon phase obtained under high pressure with shear deformation from single-wall nanotubes HiPCO, Physica B 2006; 382(1-2): 58-64.

[44] T. Michel, L. Alvarez, J. Sauvajol, R. Almairac, R. Aznar, O. Mathon, J. Bantignies, E. Flahaut., Structural selective charge transfer in iodinedoped carbon nanotubes, J. Phys. Chem. Sol. 2006; 67(5-6): 1190-1192.

[45] A. Merlen, P. Toulemonde, N. Bendiab, A. Aouizerat, J.-L. Sauvajol, G. Montagnac, H. Cardon, P. Petit, A. San Miguel, Raman spectroscopy of open-ended single wall carbon nanotubes under pressure: effect of the pressure transmitting medium, phys. stat. sol. (b) 2006; 243(3): 690-699.

[46] G. Morard, M. Mezouar, N. Rey, R. Poloni, A. Merlen, S. L. Floch, P. Toulemonde, S. Pascarelli, A. San-Miguel, C. Sanloup, G. Fiquet, Optimization of paris-edinburgh press cell assemblies for in situ monochro- 
matic x-ray diffraction and x-ray absorption, High Pressure Research 2007; 27(2): 223-233.

[47] T. Hammouda, High pressure melting of carbonated eclogite and experimental constraints on carbon recycling and storage in the mantle, Earth Planet Sci. Lett. 2003; 214(1-2): 357368.

[48] A. San-Miguel, P. Toulemonde, High-pressure properties of group IV clathrates, High Pressure Research 2005; 25(3): 159-185.

[49] M. Popov, M. Kyotani, R. J. Nemanich, Y. Koga, Superhard phase composed of single-wall carbon nanotubes, Phys. Rev. B 2002; 65(3): 033408-0334411.

[50] W. K. Wang, L. M. Cao., Transformation of carbon nanotubes to diamond at high pressure and high temperature, Russian Physics Journal 2001; 44(2): 178-182.

[51] H. Sumiya, H. Yusa, T. Inoue, H. Ofuji, T. Irifune, Conditions and mechanism of formation of nano-polycrystalline diamonds on direct transformation from graphite and non-graphitic carbon at high pressure and temperature, High Pressure Research 2006; 26(2): 63-69.

[52] A. M. Rao, P. C. Eklund, J.-L. Hodeau, L. Marques, M. N. nez Regueiro, Infrared and Raman studies of pressure-polymerized $\mathrm{C}_{60} \mathrm{~s}$, Phys. Rev. B 1997; 55(7): 4766-4773.

[53] L. Marques, M. Mezouar, J.-L. Hodeau, M. N. nez Regueiro, N. R. Serebryanaya, V. A. Ivdenko, V. D. Blank, G. A. Dubitsky, "Debye- 
Scherrer ellipses" from 3D fullerene polymers: An anisotropic pressure memory signature, Science 1999; 283: 1720-1723.

[54] S. Yamanaka, A. Kubo, N. Kini, K. Inumaru, An attempt to prepare carbon clathrate compounds using high-pressure and high-temperature conditions, Physica B 2006; 383(1): 5962.

[55] M. Nunez-Regueiro, P. Monceau, J. L. Hodeau., Crushing $\mathrm{C}_{60}$ to diamond at room-temperature, Nature 1992; 355:237-239.

[56] S. Guerini, V. Lemos, P. Piquini, S. Coutinho, Energetic and electronic properties of bn nanotube bundle under pressure, physica status solidi (b) $2007 ; 244(1): 110-115$. 


\begin{tabular}{c|c|c|c|c}
\hline Sample & $\begin{array}{c}\mathrm{P}_{\text {Max }}, \mathrm{T}_{\text {Max }} \\
(\mathrm{GPa}, \mathrm{K})\end{array}$ & PTM & Device & Obtained sample \\
\hline \hline SWCNT MER & 19,300 & Argon & DAC & $\begin{array}{c}\text { SWCNT } \\
\text { (mostly reversible) }\end{array}$ \\
\hline SWCNT MER & 24,300 & Paraffin oil & DAC & $\begin{array}{c}\text { SWCNT } \\
\text { (attenuated Raman signal) }\end{array}$ \\
\hline SWCNT NL & 12,300 & No & P-E & $\begin{array}{c}\text { SWCNT partly graphitized. } \\
\text { (SWCNT Raman still detected) }\end{array}$ \\
\hline SWCNT NL & 12,1500 & No & P-E & Disordered graphite \\
\hline SWCNT NL & $14.5,1800$ & No & Multianvil & Diamond \\
\hline I@SWCNT NL & $14.5,1800$ & No & Multianvil & Diamond \\
\hline
\end{tabular}

Table 1: Summary of high pressure and high temperature experimental conditions and obtained results. PTM is the pressure transmitting medium used if any. DAC holds for diamond anvil cell and P-E for Paris-Edinburgh cell. See text for more details.

Figure 1: Raman spectra of SWCNT (from MER corporation) before and after high pressure cycle in a diamond anvil cell $\left(\lambda_{\text {excit }}=514.5 \mathrm{~nm}\right)$. All those spectra were recorded at ambient pressure. The maximum pressure reached and the choice of the pressure transmitting medium is reported.

Figure 2: Left part: Raman spectra of SWCNT (from Nanoledge) before and after high pressure cycle in a Paris Edimburg press $\left(\lambda_{\text {excit }}=514.5 \mathrm{~nm}\right)$. The Raman spectra of disordered graphite is also reported. Right part: TEM pictures of same nanotubes submitted to high pressure (lower picture) and high pressure high temperature (upper picture) conditions. 
Figure 3: Left: Raman spectra of diamond synthesized under HP-HT (14.5 GPa $\left.1500^{\circ} \mathrm{C}\right)$ from pristine SWCNT $\lambda_{\text {excit }}=514.5 \mathrm{~nm}$, Right: SEM picture of the recovered diamond micro-crystallites.

Figure 4: Left: Raman spectrum of diamond synthetised under HP-HT (14.5 GPa 1800 K) from iodine doped SWCNT $\lambda_{\text {excit. }}=514.5 \mathrm{~nm}$, Inset: Raman spectrum of Nanoledge SWCNT, Top Right: SEM pictures of the diamond crystallites Bottom Right: High Resolution TEM picture of a single diamond crystallite.

Figure 5: Proposed schematic evolution of SWCNT bundles under high pressure and high temperature. In all cases, the arrows show the direction of combined increase of pressure and temperature. a) For large diameter tubes, pressure leads to the tubes collapse and graphitization. With increasing pressure and temperature the graphene planes are transformed into diamond.b) For tubes with a diameter small enough to prevent collapse, spatially incoherent $\mathrm{sp}^{3}$ polymerization can be expected under high pressure and high temperature. c) For bundles with mixed diameters, a possible interesting scenario could include the frustration of diamond synthesis leading to the formation of a new carboncarbon composite (see text for details). 


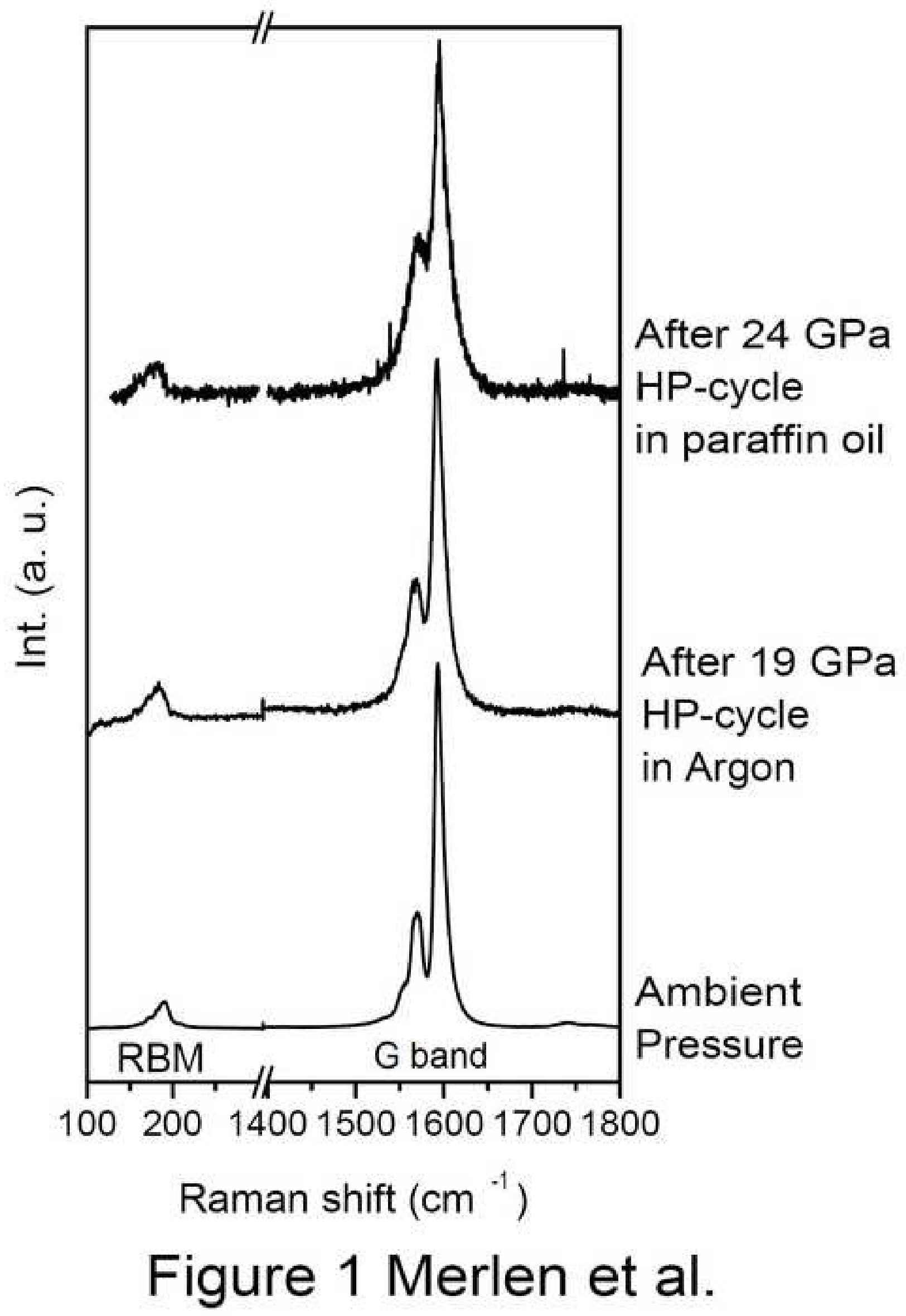




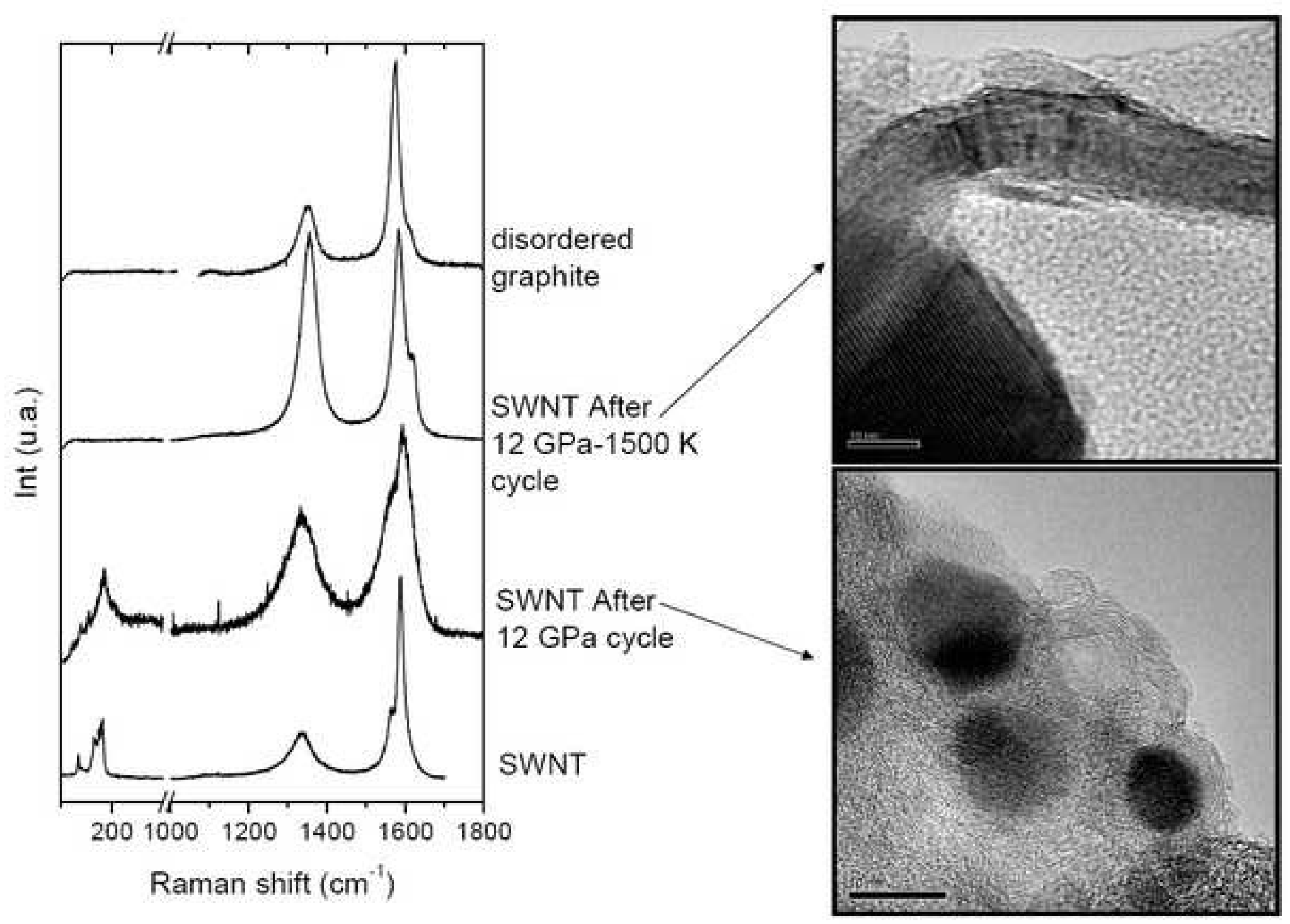

Figure 2 Merlen et al. 

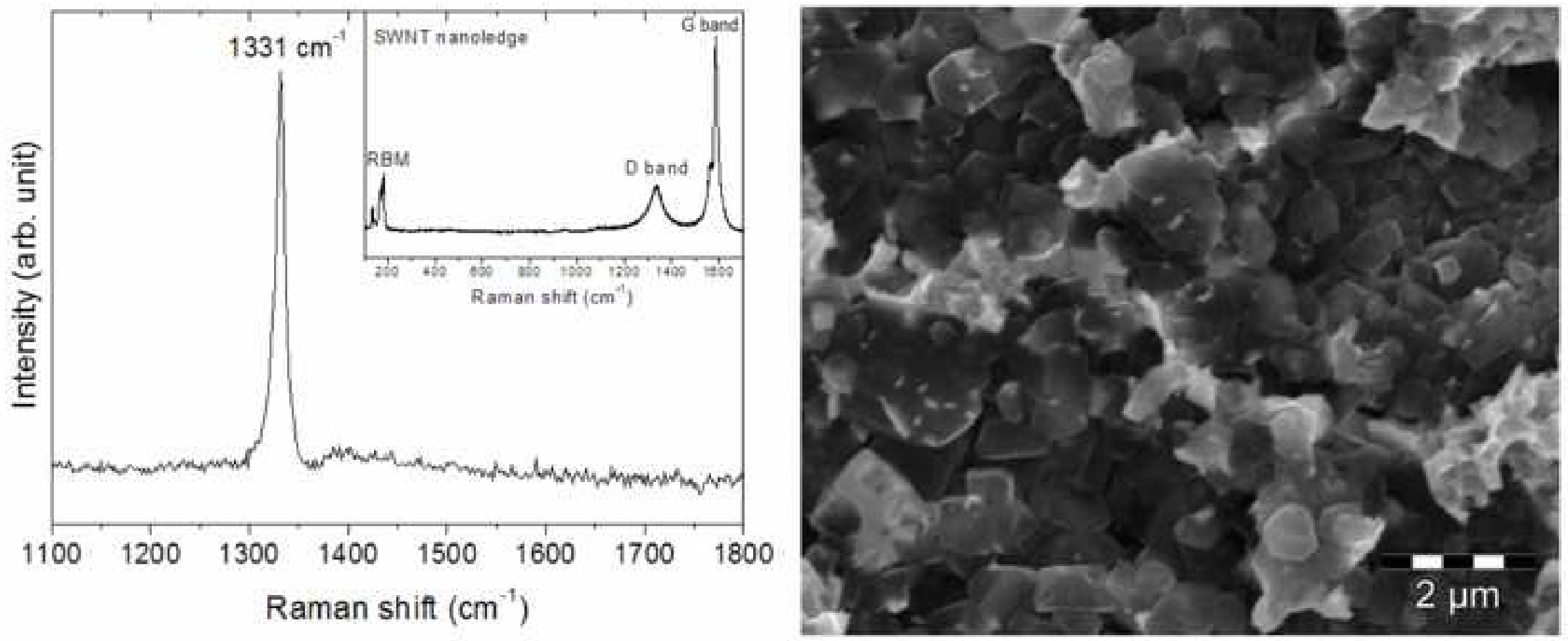

Figure 3 Merlen et al. 

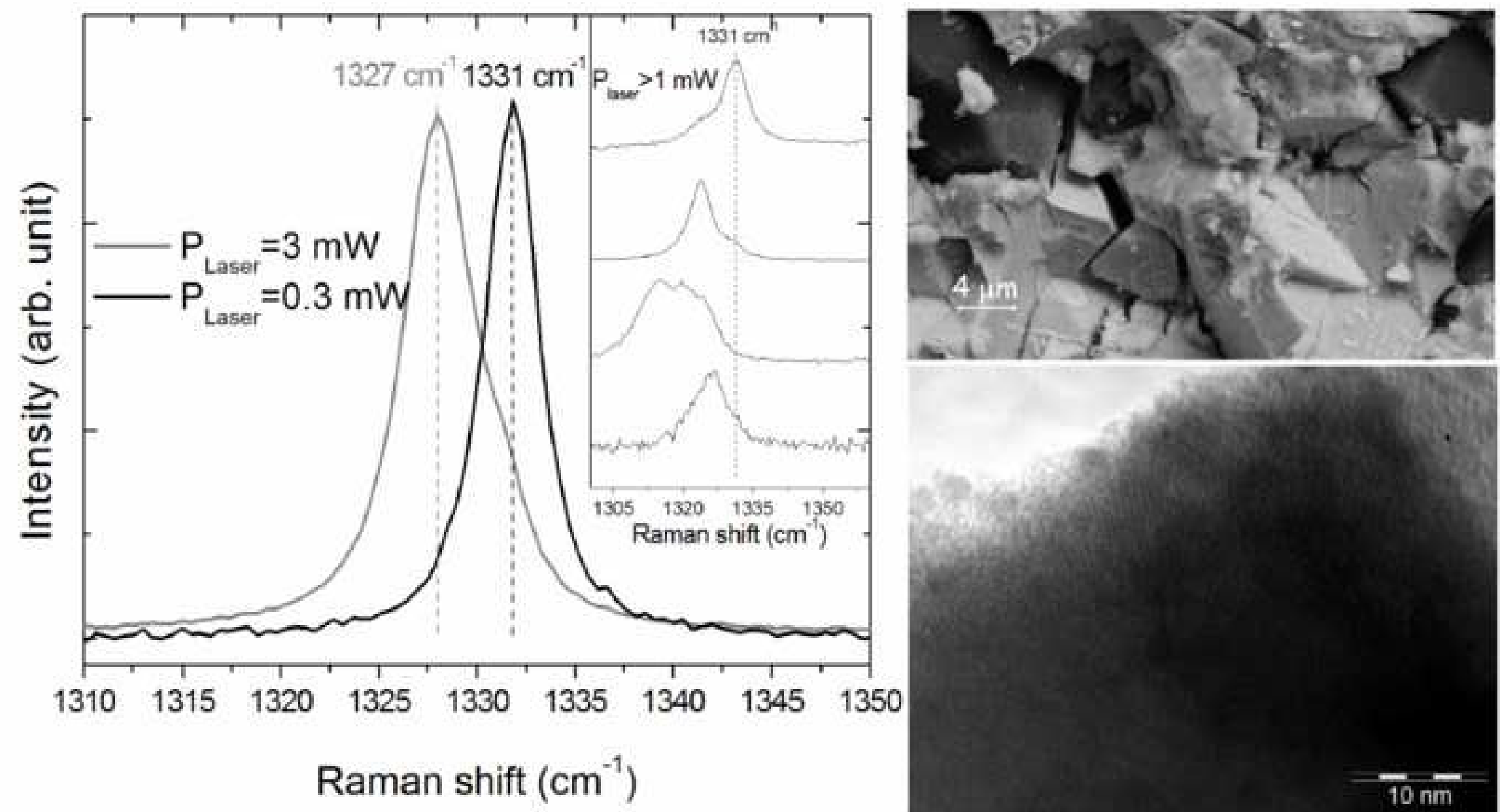

Figure 4 Merlen et al. 


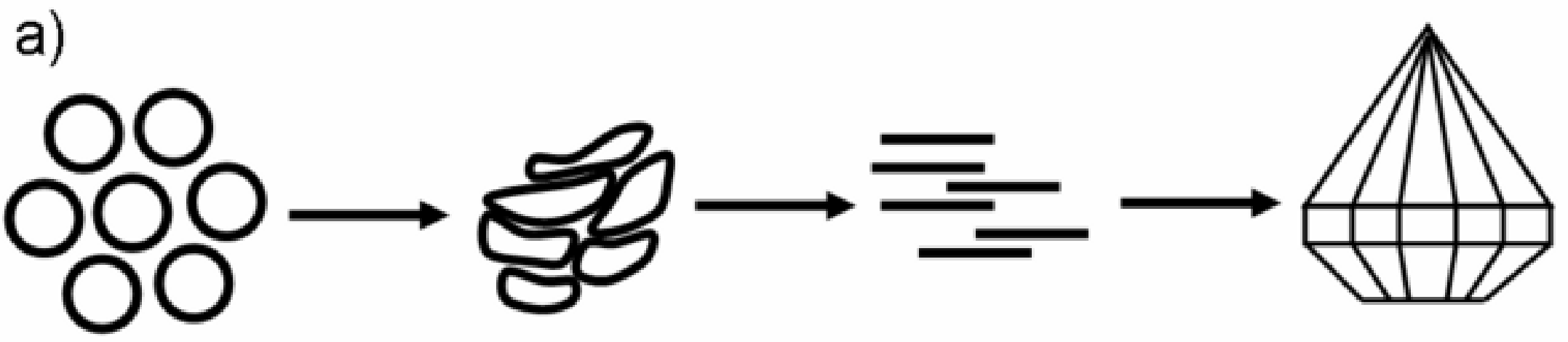

b)

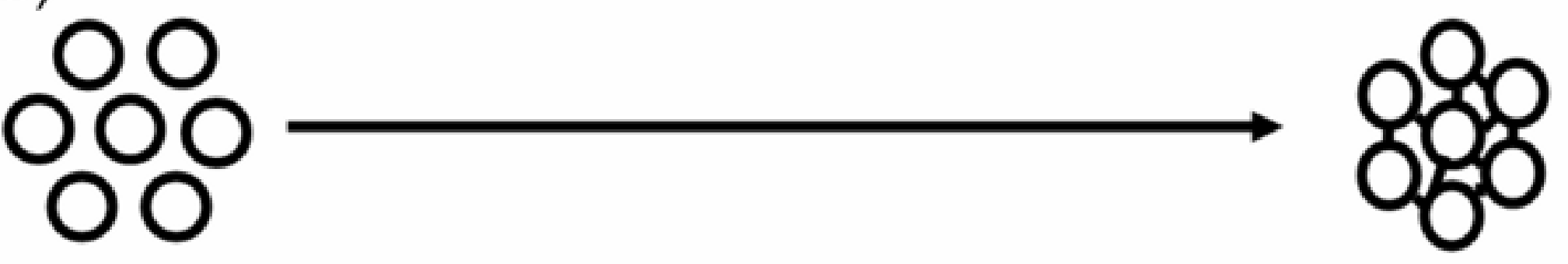

c)

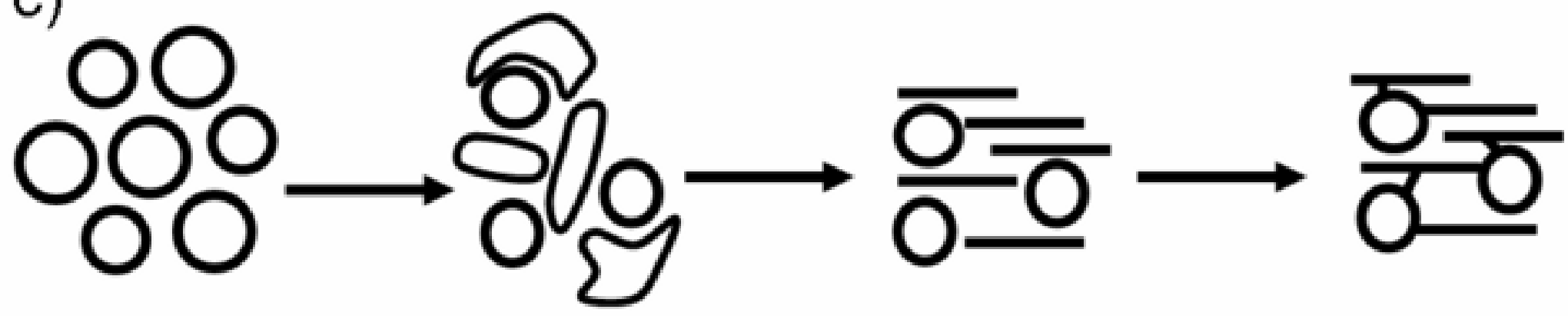

Figure 5 Merlen et al 